\title{
Paradigm shift in management of acute iatrogenic colonic perforations: 24-year retrospective comprehensive study
}

\section{(ㄷ)(1) $\ominus$}

\begin{abstract}
Authors
Institution

Department of Gastroenterology, Benizelion General

Hospital, Heraklion-Crete, Greece
\end{abstract}

Gregorios A. Paspatis, Maria Fragaki, Magdalini Velegraki, Afroditi Mpitouli, Pinelopi Nikolaou, Georgios Tribonias, Evangelos Voudoukis, Konstantinos Karmiris, Angeliki Theodoropoulou, Emmanouil Vardas

submitted 25.11 .2020

accepted after revision 11.2.2021

\section{Bibliography}

Endosc Int Open 2021; 09: E874-E880

DOI 10.1055/a-1396-4086

ISSN 2364-3722

(c) 2021. The Author(s).

This is an open access article published by Thieme under the terms of the Creative Commons Attribution-NonDerivative-NonCommercial License, permitting copying and reproduction so long as the original work is given appropriate credit. Contents may not be used for commercial purposes, or adapted, remixed, transformed or built upon. (https://creativecommons.org/licenses/by-nc-nd/4.0/)

Georg Thieme Verlag KG, Rüdigerstraße 14,

70469 Stuttgart, Germany

\section{Corresponding author}

G. A. Paspatis, MD, Benizelion General Hopsital, Department of Gastroenterology, L.Knossou, Heraklion, Crete, Greece, 71409

gpaspatis@gmail.com

Supplementary material is available under

https://doi.org/10.1055/a-1396-4086

\section{ABSTRACT}

Background and study aims Through advanced endoscopic clipping techniques, endoscopic treatment of both diagnostic and therapeutic acute iatrogenic colonic perforations has been shown effective. The main purpose of this study was to compare the management of acute iatrogenic perforations (AIPs) of the colon before and after the introduction of advanced clipping techniques.

Methods We conducted a retrospective study from July 1996 to February 2020. The period was divided into two sub periods, Period 1: from July 1996 to December 2012 and Period 2: from January 2013 to March 2020. All AIPs occurring during a colonoscopy and detected during or immediately ( $<4$ hours) after the procedure, were included in the study.

Results The total number of colonoscopies performed at our hospital was 33055 and 36831 during Periods 1 and 2 respectively. Fifteen perforations were observed in Period 1 and 11 in Period 2. The rate of surgery was $93.3 \%$ \% (14/ $15)$ in Period 1 and $27.2 \%(3 / 11)$ in Period $2(P<0.01)$. The mean hospital stay in Period 1 was 6.9 days and 4 in Period 2 $(P<0.01)$.

Conclusions Data from this historical cohort have clearly shown a decrease in the surgery rate and the length of hospitalization of AIPs in Period 2 compared to Period 1.

\section{Introduction}

Even though colonoscopy is considered to be a safe procedure, a number of serious complications such as acute iatrogenic perforations (AIPs), however small, may carry risks which are of great concern to endoscopists [1]. Indeed, having to face a new complication during treatment of the original case can be overwhelming. AIPs have been reported in $0.03 \%$ to $0.08 \%$ of diagnostic colonoscopies [2-7]. Therapeutic colonoscopy carries a greater risk of AIPs, particularly following advanced procedures $[8,9]$. In particular, two meta-analyses have reported AIPs rates of $0.9 \%$ and $1.1 \%$ following endoscopic mucosal resection (EMR) in the colon $[10,11]$.
Historically, in most cases, colonoscopic perforation has been treated surgically with laparotomy or, as is usually the case today, laparoscopically. With advanced endoscopic clipping techniques being developed during the last decade (through-the- scope [TTS] clips, with jaw distance reaching 16 $\mathrm{mm}$, a combination of TTS clips with endoloops and over-thescope-clip [OTSC system; Ovesco Endoscopy AG, Tubingen, Germany), endoscopic treatment of AIPs in both diagnostic and therapeutic procedures has been effective, particularly in perforations diagnosed during the procedure.

In the largest observational case series in the United Kingdom describing post-perforation outcomes, TTS clips were successfully used to close defects in $83.3 \%$ of cases where the per- 
foration was visualized by the endoscopist [5]. In the first prospective trial evaluating the efficacy of endoscopic closure of AIPs of the gastrointestinal tract using the OTSC system [12], authors suggested that the OTSC system might lead to a paradigm shift in the management of these patients, probably serving as a first-line endoscopic treatment. A case series evaluating AIPs enforced this assumption [13]. However, data concerning advanced clipping techniques in terms of success rate, complications, and location of perforation remain limited.

In this retrospective study, the primary objective was to compare the management of AIPs in the colon (surgery vs. endoscopic treatment) before and after the introduction of advanced clipping techniques. The secondary objectives included the evaluation of mortality, hospital stay period, and the success of clip deployment both from a technical and clinical perspective. To the best of our knowledge, this is one of the few studies evaluating the management of AIPs in the colon in a large public hospital over a 24-year period.

\section{Methods}

We conducted a retrospective study from July 1996 to February 2020. Data from all colonoscopies performed at the Benizelion General Hopspital were analyzed using the electronic database of the $\mathrm{Gl}$ endoscopic unit which was well maintained and continuously updated (Appendix 1). The period was divided in two sub-periods; Period 1: July 1996 to December 2012 and Period 2: January 2013 to March 2020. The two time periods were chosen taking into account the introduction of advanced clipping techniques in our department and therefore, 2013 was selected as the cut-off point year. This study was approved by the scientific committee of our hospital. A signed written consent form for this retrospective study was not required.

All AIPs occurring during a colonoscopy and detected during or immediately (<4 hous) after the procedure, were included in the study. Delayed perforations ( $>4$ hours after the procedure) $[1,14]$ were excluded. Such cases were excluded per protocol since most of them usually require immediate surgical treatment. Perforation was defined as a complete disruption of the colonic wall documented by an endoscopic view of either extraintestinal structures or the presence of gas and colonic contents outside the gastrointestinal tract detected in a abdominal computed tomography (CT) scan performed after the procedure. Cases of gas outside the gastrointestinal tract without the presence of colonic contents or definite instrumentation outside the large bowel lumen were not included. In both periods, all patients were managed by a multi-disciplinary team including endoscopists, radiologists, and surgeons.

Perforations during diagnostic colonoscopy were defined as those appearing during a colonoscopy performed for diagnostic reasons where no tissue was removed from the colon for therapeutic reasons. Perforations during therapeutic colonoscopy were defined as those occurring during a polypectomy, EMR or dilatation. Morbidity was defined as any complication associated to the treatment (surgical or endoscopic) of the perforation. Advanced clipping techniques were defined as the application of the OTSC system, multiple clipping with TTS clips, or the combined technique using TTS clips plus endoloop $[15,16]$. The choice of clipping technique was at the discretion of the endoscopist.

Technical success of clip application was the adequate endoscopic closure of the perforation followed by the absence of leakage as confirmed by a CT scan performed after the procedure. The absence of complications related to clip application were defined as clinical success of clip application.

The presence of colonic contents detected in an abdominal $\mathrm{CT}$ performed after the procedure, the failure of endoscopic treatment, the inadequate colon preparation and the deterioration of patient's clinical condition were the parameters taken into account by the multidisciplinary team for making the decision to treat the patient surgically $[1,17]$. The protocol briefly included surgical or endoscopic treatment in those cases where either extraintestinal structures were observed, or the presence of gas and colonic contents outside the gastrointestinal tract were detected in an abdominal CT scan, performed after the procedure. In each case the committed team decided at its discretion.

All colonoscopies were performed by trained endoscopists (no trainees were involved). In colonoscopies performed after 2012 the Boston Bowel Preparation Scale [18] was recorded. All colonoscopies were performed with the technique of one operator, with conscious sedation. After 2012, all endoscopic procedures in our department are performed using CO2. Position changes of the patient were performed when it was needed. In cases where the perforation was treated surgically, the surgeon was asked to measure the length of perforation. In cases where the perforation was treated endoscopically, perforation length was measured by visual comparison using open biopsy forceps of predetermined width or/and the diameter of the colonoscope.

\section{Statistical analysis}

Continuous data were compared with unpaired t or Mann Whitney tests as appropriate. Categorical variables were tested using corrected $\mathrm{x}^{2}$ or two-sided Fisher exact tests for univariate comparisons, as appropriate. Criterion for statistical significance was $P<0.05$.

\section{Results}

\section{Period 1}

The total number of colonoscopies performed at our hospital during Period 1 was 33055. Among them, 21375 were performed for diagnostic and 11680 for therapeutic purposes. We observed 15 perforations: 10 occurred during diagnostic and 5 during therapeutic colonoscopies ( $>$ Table 1 ). Thus, the overall iatrogenic colonic perforation rate was $0.04 \%$ ( $0.04 \%$ for diagnostic and $0.04 \%$ for therapeutic colonoscopies). Most perforations were recorded in the sigmoid colon (9/15, 60\%) and 13/ 15 (86.6\%) of patients were females. In terms of treatment, 14 patients were treated surgically and 1 endoscopically ( $>$ Table 2). The mean size of the perforation in patients having either diagnostic or therapeutic colonoscopy was $23.2 \pm 3.3$ and 11.2 $\pm 1.7 \mathrm{~mm}$, respectively. 
- Table 1 Clinical parameters of patients at study entry in the 2 studied periods.

\begin{tabular}{|l|l|l|}
\hline Variables & Period 1 & Period 2 \\
\hline Number of patients & 15 & 11 \\
\hline Sex (male) & 2 & 1 \\
\hline Age (yr) & $67.6 \pm 12.7$ & $69.1 \pm 9.5$ \\
\hline Location of perforation & & \\
\hline Rectum & 1 & 1 \\
\hline Sigmoid & 9 & 9 \\
\hline Descending & - & - \\
\hline Transverse & 1 & - \\
\hline Cecum & 4 & 1 \\
\hline Cause & & \\
\hline Diagnostic endoscopy & 10 & 7 \\
\hline Therapeutic endoscopy & 5 & 3 \\
\hline EMR & 5 & 1 \\
\hline Dilation & - & \\
\hline EMR, endoscopic mucosal resection. & & \\
\hline
\end{tabular}

\section{Surgery}

All cases were diagnosed during the procedure or immediately (<4 hours) following it. More specifically, in nine diagnostic colonoscopies, extraintestinal structures were detected during the procedure and the patients were referred for surgical treatment without any attempt for endoscopic treatment. In one therapeutic colonoscopy, a complete disruption of the colonic wall was observed during an EMR procedure. The defect was closed using six TTS clips. However, a CT scan performed immediately after the procedure revealed the presence of extraintestinal gas and colonic contents in the peritoneal cavity, thus deeming it necessary for the patient to be treated surgically. In four therapeutic cases, a CT scan was performed due to clinical signs of perforation after the completion of the procedure, showing the presence of extraintestinal gas and colonic contents, leading us to the decision of treating all of them surgically. In all surgically treated cases, a primary closure of the defect was performed with open surgery ( Table 2 and $>$ Table 3). No colonic resection was needed.

\section{TTS clips}

In one diagnostic colonoscopy, a complete disruption of the sigmoid wall was observed. The defect was closed using eight TTS clips. A CT scan performed after the procedure revealed only the presence of extraintestinal gas which, in turn, was successfully treated conservatively.
- Table 2 Treatment characteristics of patients in the two studied periods.

\begin{tabular}{|c|c|c|}
\hline Variables & $\begin{array}{l}\text { Period } 1 \\
(n=15)\end{array}$ & $\begin{array}{l}\text { Period } 2 \\
(n=11)\end{array}$ \\
\hline \multicolumn{3}{|l|}{ Treatment $^{1}$} \\
\hline Surgery & 14 & 3 \\
\hline Endoscopic treatment & 1 & 8 \\
\hline \multicolumn{3}{|l|}{ Surgery } \\
\hline Open surgery-primary closure & 14 & - \\
\hline Laparoscopic surgery-primary closure & - & 2 \\
\hline Open surgery-stoma formation & - & 1 \\
\hline Endoscopic treatment & 1 & 8 \\
\hline TTS clips & 1 & 2 \\
\hline TTS clips plus endoloop (king closure) & - & 1 \\
\hline OTSC system & - & 5 \\
\hline Failed endoscopic treatment & 1 & 2 \\
\hline $\begin{array}{l}\text { Failed endoscopic treatment with TTS } \\
\text { clips }\end{array}$ & 1 & 2 \\
\hline $\begin{array}{l}\text { Failed endoscopic treatment with OTSC } \\
\text { system }\end{array}$ & - & - \\
\hline Hospital stay (days) ${ }^{2}$ & 6.9 & 4.0 \\
\hline Mortality & 1 & - \\
\hline \multicolumn{3}{|c|}{$\begin{array}{l}\text { OTSC, over-the-scope-clip; TTS, through-the- scope. } \\
1 P<0.01 \\
2 P<0.01\end{array}$} \\
\hline
\end{tabular}

\section{Outcome}

Fourteen patients were discharged within a reasonable timeframe ( $>$ Table 2 ) as no complication was observed associated to the surgical or endoscopic treatment of the perforation. One 81-year-old woman with colon cancer and leukemia, who had been treated surgically for a perforation in the sigmoid colon during a diagnostic colonoscopy, died 2 days after surgery due to severe complications.

The technical and clinical success rates for TTS clips application were $50 \%(1 / 2)$ and $100 \%(1 / 1)$ respectively.

\section{Period 2}

Similarly, 36831 colonoscopies were performed in Period 2. Of these, 22239 were diagnostic and 14592 were therapeutic. We observed 11 perforations: seven occurred during diagnostic and four during therapeutic colonoscopies ( $>$ Table 1 ). Thus, the overall iatrogenic colonic perforation rate was $0.02 \%$ $(0.03 \%$ for diagnostic and $0.02 \%$ for therapeutic colonoscopies). Most of the perforations were observed in the sigmoid colon $9 / 11$ (81.8\%), while $10 / 11$ patients (90.9\%) were women. Regarding treatment, three patients were treated surgically and eight endoscopically ( $>$ Table 2 ). The mean size of the per- 
- Table 3 Perforations during therapeutic procedures and lesion characteristics.

\begin{tabular}{|c|c|c|c|c|c|c|}
\hline Cases & Period & Lesion & Location & Therapeutic procedure & Lesion size $(\mathrm{mm})$ & Treatment \\
\hline 1 & 1 & Sessile polyp & Transverse & EMR one piece & 15 & Open surgery-primary closure \\
\hline 2 & 1 & Sessile polyp & Cecum & EMR piecemeal & 25 & Open surgery-primary closure \\
\hline 3 & 1 & Sessile polyp & Cecum & EMR piecemeal & 20 & Open surgery-primary closure \\
\hline 4 & 1 & Sessile polyp & Cecum & EMR piecemeal & 30 & Open surgery-primary closure \\
\hline 5 & 1 & Sessile polyp & Cecum & EMR piecemeal & 30 & Open surgery-primary closure \\
\hline 6 & 2 & Sessile polyp & Cecum & EMR one piece & 15 & Laparoscopic surgery- primary closure \\
\hline 7 & 2 & Sessile polyp & Sigmoid & EMR piecemeal & 30 & Endoscopic treatment, TTS clips \\
\hline 8 & 2 & $\begin{array}{l}\text { Benign } \\
\text { stenosis }\end{array}$ & $\begin{array}{l}\text { Colorectal } \\
\text { anastomosis }\end{array}$ & $\begin{array}{l}\text { Endoscopic balloon } \\
\text { dilation }\end{array}$ & 20 & Open surgery-Stoma formation \\
\hline 9 & 2 & Sessile polyp & Sigmoid & EMR one piece & 20 & Endoscopic treatment-OTSC system \\
\hline
\end{tabular}

OTSC, over-the-scope-clip; TTS, through-the-scope; EMR, endoscopic mucosal resection.

foration in patients having either a diagnostic or therapeutic colonoscopy was $24.4 \pm 2.2$ and $18.7 \pm 4.7 \mathrm{~mm}$, respectively.

\section{OTSC system}

In four diagnostic colonoscopies, extraintestinal structures (all in the sigmoid colon) were identified during the procedure. In all patients, an OTSC system was applied and the defect was effectively closed ( $>$ Table 2 ). A CT scan performed in all cases after the application of OTSC system revealed the presence of only extraintestinal gas. In one therapeutic colonoscopy ( $>\mathrm{Ta}$ ble 3) a complete disruption of the sigmoid wall was observed during an EMR (en block resection of a 2-cm sessile polyp in the sigmoid colon). The defect was successfully closed with an OTSC and was then checked via a CT scan which revealed only the presence of extraintestinal gas. All five patients uneventfully received further conservative treatment. We did not face any complications related to the OTSC system application.

\section{TTS clips}

In two colonoscopies (one diagnostic and one therapeutic; EMR in the sigmoid colon) a complete rupture of the sigmoid wall was recognized ( $\triangleright$ Table 2 and $\triangleright$ Table 3 ). The defect was closed by applying seven and six TTS clips in each case respectively, and the CT scans performed after the procedures revealed the presence of only extraintestinal gas. Thereafter, the patients were treated conservatively without complications. Finally, in another diagnostic colonoscopy a complete disruption of the rectal wall was observed after a retroflexion manoeuvre. Perforation was effectively treated with eight TTS clips and endoloop (king closure). CT scan after the procedure showed the presence of only extraintestinal gas. Once again, the patient received further conservative treatment without complications

\section{Surgery}

In one therapeutic colonoscopy, a complete rupture of the cecum wall was identified during an EMR procedure. The defect was closed using eight TTS clips. However, the closure was not considered efficient and the patient was treated surgically. A primary closure of the defect was immediately performed laparoscopically. In another diagnostic colonoscopy, a complete disruption of the sigmoid wall was observed. The defect was closed using seven TTS clips. However, the closure was not considered adequate and the patient was referred for surgery and a primary closure of the defect was performed laparoscopically ( $>$ Table 2 and $>$ Table 3). In both cases TTS clips closure was considered incomplete since defect in the colonic wall was still observed, being not amenable to further endoscopic treatment and the decision to treat the patients surgically was made. The OTSC was not available at that time. In a third patient, a perforation occurred during endoscopic balloon dilation of a benign colorectal anastomosis stricture after surgery for adenocarcinoma in the sigmoid colon. The patient was immediately treated with open surgery with a formation of a temporal stoma.

\section{Outcome}

Eleven patients were discharged within a reasonable timeframe ( Table 2). Both laparoscopically treated patients were discharged 3 days after the operation. No complications associated with the surgical or endoscopic treatment of the perforation were observed in these patients during hospitalization.

The technical success rate for TTS clip application was $60 \%$ (3/5) including the case with the endoloop combination. The technical success rate for OTSC system application was $100 \%$ (5/5). The clinical success rate for all clipping devices was $100 \%$. The percentage of patients undergoing laparoscopic surgery was $66.6 \%(2 / 3)$.

\section{Comparison of the two periods}

The rate of surgery was $93.3 \% \%(14 / 15)$ for Period 1 , and $27.2 \%$ $(3 / 11)$ for Period $2(P<0.01)$ ( $>$ Table 2$)$ ( $>$ Fig. 1$)$. The subsequent rate of endoscopic treatment increased from $6.6 \%$ to $72.7 \%$. In the subgroup of cases where AIPs occurred during a diagnostic colonoscopy, the respective rate of endoscopic 


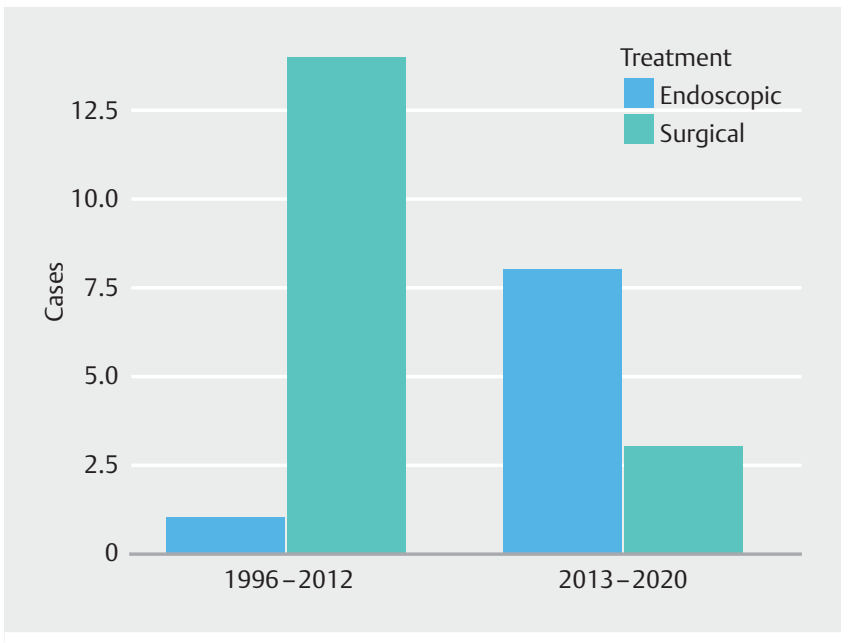

- Fig. 1 Comparison of treatment during the two time periods.

treatment increased from $0 \%$ for Period 1 to $85.7 \%$ for Period 2. The mortality rate was $8.3 \%(1 / 15)$ for Period 1 and $0 \%(0 / 11)$ for Period 2 of the study, and this difference was not significant $(P=1)$. The mean hospital stay was 6.9 days, and 4 days for Period 1 and 2 respectively $(P<0.01)$ ( $>$ Table 2 ).

The Boston Bowel Preparation Scale score was $>6$ in the cases which were amenable to be calculated.

\section{Discussion}

In this retrospective study, we primarily evaluated the management of colonic AIPs (surgery vs endoscopic treatment) between Period 1 and Period 2. A definite shift to endoscopic treatment (from $6.7 \%$ to $72.7 \%$ ) was demonstrated. Secondarily, there was a significant reduction in length of hospitalization during Period 2. It would be noteworthy to mention that the technical and clinical success rate of the OTSC system application remained $100 \%$ even in large perforations observed during a diagnostic colonoscopy $\sim 2 \mathrm{~cm}$.

AIPs related to colonoscopy is defined as the recognition of gas or colonic contents outside the colon $[1,19]$ or any clearly visible sign of perforation identified endoscopically during or in time related to endoscopy. In the present study, cases with gas outside the $\mathrm{Gl}$ tract but without the presence of colonic contents or definite instrumentation outside the large bowel lumen were not included. In other words, cases with colonic perforation were included where endoscopic or surgical treatment was mandatory because otherwise, serious complications would appear. This could explain the similar perforation rates between diagnostic and therapeutic colonoscopies.

It is well documented that the timing of diagnosis is important for the management of the AIPs of the colon and the patient outcome [20,21]. Morbidity and mortality have significantly been related to the delay of AIPs diagnosis $>24$ hours [22]. In this study, all AIPs occurring during a colonoscopy that were detected during or immediately ( $<4$ hours) after the procedure were included in the study. Therefore, the associated mortality and morbidity rates were rather low. No cases with delayed diagnosed perforations were included. After searching rigorously across our electronic database, we did not find any case of delayed colonic perforation requiring surgical treatment. As recommended, all patients in our unit are thoroughly evaluated after colonoscopy for clinical symptoms suggestive of perforation and are not allowed for discharge until they are completely free of such symptoms [1]. In cases of doubt, these patients may undergo an abdominal CT scan with the intention of detecting radiologic findings compatible with perforation. We acknowledge that we have a low threshold for performing abdominal CT scan in patients with post-colonoscopy symptoms suggesting perforation, and, by adopting this policy, the number of cases with delayed colon AIPs has practically been eliminated.

There is enough evidence that perforations occurring during diagnostic colonoscopies are larger than those occurring during therapeutic colonoscopies $[17,23,24]$ and this was confirmed in our data. The OTSC system is considered more effective in defects larger than $1 \mathrm{~cm}$, performed mainly during diagnostic colonoscopy due to its ability to capture and sustain a larger volume of tissue with a higher compression force compared to conventional TTS clips. Furthermore, relatively large defects can also be sutured by using dedicated grasping forceps. It is of the utmost importance to avoid suctioning the extraluminal structures into the cap of the OTSC system and therefore, caution is strongly recommended in the phase of pulling the wall defect to the cap via suction. Suction of extraluminal structures into the cap of the OTSC ${ }^{\circledR}$ system have sparsely been reported $[25,26]$. To minimize such a risk and to succeed in the closure of even larger defects, the use of a twin grasper has been recommended. In this study, we did not use the twin grasper device. However, we did not face any complications coming from the OTSC system application. The technical and clinical success rate of OTSC was $100 \%$, while it was mostly used in large defects performed during diagnostic colonoscopy. We could make a speculation that the extended experience in the use of OTSC system and Full Thickness Resection Device (FTRD, Ovesco Endoscopy AG, Tubingen, Germany) devices in our department [27] has resulted in the uneventful application of the OTSC system. Nevertheless, the number of presented cases treated with the OTSC system is rather small and a misjudgement could not be excluded. In other studies, a few failures concerning the technical success of the OTSC system application have been reported $[12,13,28,29]$.

The sigmoid colon and rectosigmoid junction are the most common sites of AIPs during a diagnostic procedure due to direct mechanical injury applied by the colonoscope during insertion $[5,6,23,30]$. In agreement with the existing literature, most perforations in this study occurred in the sigmoid colon, particularly those during diagnostic colonoscopy, and it is therefore difficult to make comparisons based on these data, with regard to the effectiveness of advanced clipping technique in the different segments of the colon. Indeed, there is lack of data to adequately evaluate this issue. It is worth noting that the use of the OTSC system in the right colon is related to certain technical obstacles due to the rather difficult advancement of the colonoscope mounted with the OTSC system. However, 
the experience with FTRD, a rather similar device, has assisted in overcoming obstacles concerning the advancement of the mounted colonoscope [27, 31].

In the first prospective trial evaluating the efficacy of endoscopic closure of AIPs of the GI tract using the OTSC system, Voermans et al [12] revealed a $92 \%$ success rate in the colon among the 13 cases included. Additionally, in this study the assumption that the OTSC system might lead to a paradigm shift in the management of these patients was done closing the defects with the OTSC system as a first-line treatment. Retrospective studies and reviews in $\mathrm{Gl}$ perforations have enforced these assumptions [13, 28, 29, 32]. Our study comes some years later to confirm the paradigm shift, specifically in the colon, by analyzing a large colonoscopic historical cohort in both a disgnostic and therapeutic setting. Our data revealed a significant reduction regarding length of hospitalization in patients treated endoscopically or laparoscopically, leading to the speculation that this might be associated with lower morbidity, although it was not clearly proven by these data. After a multidisciplinary approach, all patients treated endoscopically for AIPs of the coIon remained in hospital to receive intravenous antibiotic treatment despite not having developed any complications. We may assume that if this strategy had not been adopted, the difference in the length of hospitalization between the two periods would be greater.

In this study the technical and clinical success rate of TTS clipping is $50 \%$. In the largest retrospective observational case series in Europe describing post-perforation outcomes, TTS clips were successfully used to close defects in $83.3 \%$ of cases where the perforation was visualized by the endoscopist [5]. This discrepancy might be explained by the exclusion of EMR cases with type 3 and 4 mural injury (Sydney Classification of Deep Mural Injury (DMI) following EMR [33]) which are considered perfectly amenable to the TTS clipping. Indeed, only cases with defects within a white cautery ring and observed contamination were included (type 5) (Sydney Classification of DMI following EMR). Indeed, this was also applied to the Period 1 cases, where the entry criterion was defined as the presence of colonic contents outside the colon, i.e. open contamination that is not only limited to the presence of extraluminal gas which does not necessarily presuppose open perforation.

There are some limitations in our study. First, this study is a retrospective one and it was performed in the setting of one center, thus the results cannot be easily generalized. Second, the number of patients included is small. However, this limitation is unavoidable since perforation is not a frequent complication of colonoscopy. Finally and most importantly, it is difficult to make accurate comparisons with previous studies, and in particular comparisons concerning the perforation rates, since confounding factors are created by variations in methodology as well as by differences in the definition of the perforation. The strength of this study is that the database in our unit was well maintained, continuously updated and resulted in minimizing the possibility of underestimating the cases of perforation and the subsequent required treatment.

\section{Conclusions}

In conclusion, data from this historical cohort study have clearly shown a decrease in the surgery rate of AIPs occurring in both diagnostic and therapeutic colonoscopies and detected during or immediately after the procedure. In addition, after the endoscopic or laparoscopic treatment of AIPs, a significant reduction in the required days of hospitalization was observed.

\section{Competing interests}

The authors declare that they have no conflict of interest.

\section{References}

[1] Paspatis GA, Arvanitakis M, Dumonceau JM et al. Diagnosis and management of iatrogenic endoscopic perforations: European Society of Gastrointestinal Endoscopy (ESGE) Position Statement - Update 2020. Endoscopy 2020; 52: 792-810

[2] Stock C, Ihle P, Sieg A et al. Adverse events requiring hospitalization within 30 days after outpatient screening and nonscreening colonoscopies. Gastrointest Endosc 2013; 77: 419-429

[3] Lee T], Rutter MD, Blanks RG et al. Colonoscopy quality measures: experience from the NHS Bowel Cancer Screening Programme. Gut 2012; 61: 1050-1057

[4] Rabeneck L, Saskin R, Paszat LF. Onset and clinical course of bleeding and perforation after outpatient colonoscopy: a population-based study. Gastrointest Endosc 2011; 73: 520-523

[5] Derbyshire E, Hungin P, Nickerson C et al. Colonoscopic perforations in the English National Health Service Bowel Cancer Screening Programme. Endoscopy 2018; 50: 861-870

[6] Paspatis GA, Vardas E, Theodoropoulou A et al. Complications of colonoscopy in a large public county hospital in Greece. A 10-year study. Digest Liver Dis 2008; 40: 951-957

[7] Paraskeva KD, Paspatis GA. Management of bleeding and perforation after colonoscopy. Expert Rev Gastroenterol Hepatol 2014; 8: 963972

[8] Takamaru H, Saito Y, Yamada M et al. Clinical impact of endoscopic clip closure of perforations during endoscopic submucosal dissection for colorectal tumors. Gastrointest Endosc 2016; 84: 494-502.e491

[9] Taku K, Sano Y, Fu KI et al. latrogenic perforation associated with therapeutic colonoscopy: a multicenter study in Japan. J Gastroenterol Hepatol 2007; 22: 1409-1414

[10] Arezzo A, Passera R, Marchese N et al. Systematic review and meta-analysis of endoscopic submucosal dissection vs endoscopic mucosal resection for colorectal lesions. United Europ Gastroenterol J 2016; 4: $18-29$

[11] Kothari ST, Huang RJ, Shaukat A et al. ASGE review of adverse events in colonoscopy. Gastrointest Endosc 2019: doi:10.1016/j.gie.2019. 07.033

[12] Voermans RP, Le Moine O, von Renteln D et al. Efficacy of endoscopic closure of acute perforations of the gastrointestinal tract. Clin Gastroenterol Hepatol 2012; 10: 603-608

[13] Khater S, Rahmi G, Perrod G et al. Over-the-scope clip (OTSC) reduces surgery rate in the management of iatrogenic gastrointestinal perforations. Endosc Int Open 2017; 5: E389-E394

[14] Carr-Locke DL. The changing management of colonoscopy-associated perforations. Digestion 2008; 78: 216-217 
[15] Martinek J, Ryska O, Tuckova I et al. Comparing over-the-scope clip versus endoloop and clips (KING closure) for access site closure: a randomized experimental study. Surg Endosc 2013; 27: 1203-1210

[16] Shi Q, Chen T, Zhong YS et al. Complete closure of large gastric defects after endoscopic full-thickness resection, using endoloop and metallic clip interrupted suture. Endoscopy 2013; 45: 329-334

[17] Cho SB, Lee WS, Joo YE et al. Therapeutic options for iatrogenic colon perforation: feasibility of endoscopic clip closure and predictors of the need for early surgery. Surg Endosc 2012; 26: 473-479

[18] Lai E], Calderwood AH, Doros G et al. The Boston bowel preparation scale: a valid and reliable instrument for colonoscopy-oriented research. Gastrointest Endosc 2009; 69: 620-625

[19] Cotton PB, Eisen GM, Aabakken L et al. A lexicon for endoscopic adverse events: report of an ASGE workshop. Gastrointest Endosc 2010; 71: 446-454

[20] Raju GS, Saito Y, Matsuda T et al. Endoscopic management of colonoscopic perforations (with videos). Gastrointest Endosc 2011; 74: $1380-1388$

[21] Baron TH, Wong Kee Song LM, Zielinski MD et al. A comprehensive approach to the management of acute endoscopic perforations (with videos). Gastrointest Endosc 2012; 76: 838-859

[22] La Torre M, Velluti F, Giuliani $G$ et al. Promptness of diagnosis is the main prognostic factor after colonoscopic perforation. Colorectal Dis 2012; 14: e23-e26

[23] Hawkins AT, Sharp KW, Ford MM et al. Management of colonoscopic perforations: A systematic review. Am J Surgery 2018; 215: 712-718

[24] Teoh AY, Poon CM, Lee JF et al. Outcomes and predictors of mortality and stoma formation in surgical management of colonoscopic perforations: a multicenter review. Arch Surgery 2009; 144: 9-13
[25] Loske G, Schorsch T, Daseking E et al. Small intestine grasped by overthe-scope-clip during attempt to close an iatrogenic colonic perforation. Endoscopy 2016; 48: E26-E27

[26] Rahmi G, Barret M, Samaha E et al. Ureteral obstruction after colonoscopic perforation closed with an over-the-scope clip. Gastrointest Endosc 2015; 81: 470-471; discussion 471-172

[27] Velegraki M, Trikola A, Vasiliadis K et al. Endoscopic full-thickness resection of colorectal lesions with the full-thickness resection device: clinical experience from two referral centers in Greece. Ann Gastroenterol 2019; 32: 482-488

[28] Haito-Chavez Y, Law JK, Kratt T et al. International multicenter experience with an over-the-scope clipping device for endoscopic management of GI defects (with video). Gastrointest Endosc 2014; 80: 610-622

[29] Horenkamp-Sonntag D, Liebentraut J, Engel S et al. Use of over-thescope clips in the colon in clinical practice: results from a German administrative database. Endoscopy 2020: doi:10.1055/a-1206-0778

[30] Luning TH, Keemers-Gels ME, Barendregt WB et al. Colonoscopic perforations: a review of 30,366 patients. Surg Endosc 2007; 21: 994-997

[31] Schmidt A, Beyna T, Schumacher B et al. Colonoscopic full-thickness resection using an over-the-scope device: a prospective multicentre study in various indications. Gut 2018; 67: 1280-1289

[32] Kobara H, Mori H, Nishiyama $\mathrm{N}$ et al. Over-the-scope clip system: A review of 1517 cases over 9 years. J Gastroenterol Hepatol 2019; 34: 22-30

[33] Burgess NG, Bassan MS, McLeod D et al. Deep mural injury and perforation after colonic endoscopic mucosal resection: a new classification and analysis of risk factors. Gut 2017; 66: 1779-1789 